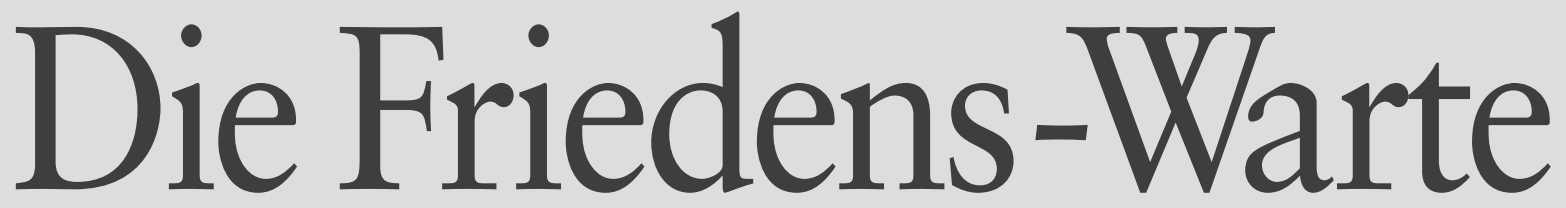

Sonder-

druck
Vol. 93 | 2020 | Issue 3-4

Christian Tomuschat zu Ehren

Honouring

Christian Tomuschat

Mit Beiträgen von

DANIEL-ERASMUS KHAN

CHRISTINA BINDER

CHRISTIAN WALTER/PHILIP NEDELCU

CHRISTIAN J. TAMS

OLIVER DIGGELMANN

BARDO FASSBENDER

ERIKA DE WET

RÜDIGER LÜDEKING/HELMUT W. GANSER

ZABRINA WELTER/CHRISTINA ANKEN-

BRAND

BENEDIKT BEHLERT 



\title{
Die Beharrungskraft des Status quo: Die Bemühungen um eine Reform des Sicherheitsrates im Jahr des 75-jährigen Bestehens der Vereinten Nationen
}

\author{
BARDO FASSBENDER
}

\begin{abstract}
Seit nunmehr fast dreißig Jahren diskutieren die Mitgliedstaaten der UN eine grundlegende Reform der Zusammensetzung und der Abstimmungsregeln des Sicherheitsrats. Im Mittelpunkt der Kontroverse stehen eine Erweiterung des Rats um zusätzliche ständige und nichtständige Mitglieder sowie das Problem des Vetorechts der ständigen Mitglieder. Eine Einigung konnte bisher nicht erzielt werden. Gleichwohl halten die Mitgliedstaaten am Ziel einer Reform dieses für die UN, aber auch für die Völkerrechtsordnung als Ganzes zentral wichtigen Organs fest, die seine Legitimität und Effektivität erhöhen würde. Der Beitrag skizziert den Verlauf der Reformdebatte seit 1991 und ihren gegenwärtigen Stand. An seinem Schluss stehen der Versuch einer Zwischenbilanz und ein Blick in die mögliche Zukunft des schwierigen Reformprojekts.
\end{abstract}

Keywords UN-Reform, UN-Sicherheitsrat, Vetorecht, Multilateralismus, Zukunft der Vereinten Nationen

The Persistence of the Status quo:

The Efforts to Reform the UN Security Council in the Year of the 75th Anniversary of the United Nations

\begin{abstract}
For almost thirty years, UN member states have been discussing a fundamental reform of the membership and the voting procedure of the Security Council. The focus of the controversy is on an expansion of the Council by adding new permanent and non-permanent members, and on the problem of the veto power of the permanent members. So far, an agreement could not be reached. Nevertheless, member states hold on to the aim of reforming the Council, as an organ of central importance not only of the UN but of the international legal order as a whole, with a view to enhancing its legitimacy and effectiveness. This article gives a summary of the reform discussion as it has taken place since 1991, and of its present status. It concludes by drawing up an interim balance of the debate, and by casting a glance at the possible future of the difficult project of Security Council reform.
\end{abstract}

Keywords UN reform; UN Security Council; veto power; mulilateralism; future of the United Nations 


\section{Die Erklärung der Generalversammlung vom September 2020}

Zur Feier des 75-jährigen Bestehens der Vereinten Nationen haben die Staats- und Regierungschefs der UN-Mitgliedstaaten in einer virtuellen Sitzung der Generalversammlung am 21. September 2020 eine „Declaration on the commemoration of the seventy-fifth anniversary of the United Nations“ angenommen (Resolution 75/1). Zur Frage der Reform des UN-Sicherheitsrates enthält die Deklaration nur einen Halbsatz: „We commit to instil new life in the discussions on the reform of the Security Council".. ${ }^{1}$ Dieser befindet sich im zweiten Teil der Erklärung, der mit dem Absatz 7 beginnt und aus zwölf in jeweils einem Absatz formulierten Handlungsversprechen besteht, in dem mit den Worten „We will upgrade the United Nations" beginnenden Absatz 14. Wahrscheinlich sollen diese dürren Worte ungefähr dem entsprechen, was vor fünf Jahren, in der Erklärung der Generalversammlung zum siebzigsten Bestehen der Vereinten Nationen, so ausgedrückt wurde: „We bear in mind the ongoing process of United Nations reforms in all their aspects." 2 Man kann die Formulierung „to instil new life in the discussions“ aber auch anders lesen: Wem vorsichtig und langsam neues Leben eingeflößt werden muss, so wie dem Schwerkranken tröpfchenweise eine Medizin, der befindet sich in sehr schlechter Verfassung, ja liegt vielleicht schon im Sterben. Und tatsächlich sind um das Krankenbett der Sicherheitsratsreform seit Jahren nur noch professionelle Pflegekräfte versammelt, die sich des vor sich hin Dämmernden von Berufs wegen mit einem Minimum von Energie und Aufwand annehmen. Besuche von Verwandten und Freunden sind sehr selten geworden, und wenn sie stattfinden, beschränken sie sich auf wenige Minuten, in denen der Kranke mit einigen aufmunternden Floskeln bedacht wird. Mancher auf der Station wartet auf den Chefarzt, der entschlossen anordnet, den Tropf abzustellen, aber auch dieser lässt sich nicht blicken.

Wegen der Covid-19-Pandemie fanden in New York seit März 2020 über die Sicherheitsratsreform keine Diskussionen mehr im Rahmen der Intergovernmental Negotiations ${ }^{3}$ der Generalversammlung statt. Nach zwei Sitzungen am 13./14. Februar 2020 (über das weitere Prozedere) und 4./5. März 2020 (über das Themenbündel „,regional representation“) mussten die für Ende März und Ende April geplanten weiteren Sitzungen ${ }^{4}$ abgesagt werden. ${ }^{5}$ Es gelang den beiden Ko-Vorsitzenden der Intergovernmental Negotiations in der 74. Sitzungsperiode der Generalversammlung auch nicht, ein Einvernehmen der Mitgliedstaaten über eine Fortsetzung der Besprechungen in digitalen Übertragungsformen zu erzielen. ${ }^{6}$ Daher wird es gemäß der „roll-over decision“ 74/569 der Generalversammlung vom 31. August $2020^{7}$ erst im Verlauf der 75. Sitzungsperiode zu weiteren Beratungen über die Frage der Sicherheitsratsreform kommen.

Wie anders und inhaltsreicher als im Jahr 2020, wenngleich auch schon formelhaft hatte es noch 2005 geklungen, als sich die Staats- und Regierungschefs der Mitgliedstaaten in

1 Vgl. zur Entstehung der Deklaration unter den Bedingungen der Covid-19-Pandemie: UNO, Generalversammlung 2020a.

2 UNO, Generalversammlung 2015, Abs. 8.

3 Vgl. unten Abschnitt 2.5.

4 Vgl. für die vorgesehenen Themen den Arbeitsplan der Ko-Vorsitzenden: UNO, Generalversammlung 2019d, sowie UNO, Generalversammlung 2020c.

5 Vgl. UNO, Generalversammlung 2020d.

6 Vgl. UNO, Generalversammlung 2020e.

7 UNO, Generalversammlung $2020 \mathrm{f}$. 
ihrem World Summit Outcome im Abschnitt „Strengthening the United Nations“ auf folgende Formulierung zur Sicherheitsratsreform verständigten:

We support early reform of the Security Council - an essential element of our overall effort to reform the United Nations - in order to make it more broadly representative, efficient and transparent and thus to further enhance its effectiveness and the legitimacy and implementation of its decisions. We commit ourselves to continuing our efforts to achieve a decision to this end and request the General Assembly to review progress on the reform set out above by the end of $2005 .^{8}$

Fünf Jahre zuvor war in der United Nations Millennium Declaration noch die Rede von einer ,umfassenden Reform des Sicherheitsrates“ die Rede gewesen („We resolve therefore: [...] To intensify our efforts to achieve a comprehensive reform of the Security Council in all its aspects $\left.{ }^{69}\right)$.

In keiner dieser Erklärungen ist die Generalversammlung jedoch auf das konkrete Ziel einer Erweiterung des Sicherheitsrates um zusätzliche Mitglieder zurückgekommen, zu dem sie sich in der Erklärung zum 50-jährigen Bestehen der UN im Oktober 1995 bekannt hatte:

In order to be able to respond effectively to the challenges of the future and the expectations of the United Nations held by peoples around the world, it is essential that the United Nations itself be reformed and modernized. [...] The Security Council should, inter alia, be expanded and its working methods continue to be reviewed in a way that will further strengthen its capacity and effectiveness, enhance its representative character and improve its working efficiency and transparency; as important differences on key issues continue to exist, further in-depth consideration of these issues is required. [...] These and other changes, within the United Nations system, should be made if we are to ensure that the United Nations of the future serves well the peoples in whose name it was established. ${ }^{10}$

Die Erklärung von 1995 nahm in ihrer Präambel ausdrücklich Bezug auf das Ende des Kalten Krieges, einen wesentlichen, inzwischen fast vergessenen und jedenfalls in der Diskussion heute nicht mehr erwähnten Impetus für eine Reform des Sicherheitsrates:

At this time, following the end of the cold war, and as the end of the century approaches, we must create new opportunities for peace, development, democracy and cooperation. The speed and extent of change in today's world point to a future of great complexity and challenge and to a sharp increase in the level of expectations of the United Nations. ${ }^{11}$

8 UNO, Generalversammlung 2005, Abs. 153.

9 UNO, Generalversammlung 2000, Abs. 30, Unterabs. 2.

10 UNO, Generalversammlung 1995, Abs. 14 (unter Weglassung der Ausführungen über die Generalversammlung und den Wirtschafts- und Sozialrat).

11 UNO, Generalversammlung 1995, Präambel, Abs. 3. 


\section{Die Reformdebatte in Grundzügen und Höhepunkten}

\subsection{Der Beginn der Debatte im Jahr 1991 und die Hauptanliegen der Verfechter einer Reform des Sicherheitsrates}

Begonnen hatte die Debatte am Sitz der UN über eine Reform des Sicherheitsrates mit Beratungen im Plenum der Generalversammlung im Dezember 1991 über den Tagesordnungspunkt „Question of equitable representation on and increase in the membership of the Security Council“. ${ }^{12}$ Dieser war seit 1979 Teil der Tagesordnung der Generalversammlung gewesen, seit dem folgenden Jahr aber nicht mehr diskutiert, sondern von Jahr zu Jahr vertagt worden. Im Jahr 1979 hatte eine von Indien angeführte Gruppe von Mitgliedstaaten vorgeschlagen, die Zahl der nichtständigen Mitglieder des Sicherheitsrates von zehn auf vierzehn zu erhöhen. Ein Resolutionsentwurf lateinamerikanischer Staaten sah eine Erhöhung auf sechzehn nichtständige Sitze vor. Beide Initiativen scheiterten an der Ablehnung durch alle ständigen Mitglieder des Sicherheitsrates, ausgenommen nur China. ${ }^{13}$

Auch im Jahr 1991 eröffneten Vertreter Indiens und Mexikos die Debatte. Beteiligten sich 1991 acht Staaten an der Diskussion (darunter nur ein westlicher Staat, Italien), waren es im folgenden Jahr bereits sechzehn. ${ }^{14}$ In der Zwischenzeit hatte sich die Bewegung der blockfreien Staaten (NAM) im September 1992 in Jakarta für eine Reform des Sicherheitsrates ausgesprochen. ${ }^{15}$ Im Ergebnis der Debatte von 1992 nahm die Generalversammlung Resolution 47/62 an, mit der sie unter anderem ,the changed international situation and the substantial increase in the membership of the United Nations“" anerkannte (Präambel, 2. Abs.) und den UN-Generalsekretär bat, die Mitgliedstaaten zu ,written comments on a possible review of the membership of the Security Council“" aufzufordern (Abs. 1). ${ }^{16}$ Als daraufhin 75 Mitgliedstaaten aus allen Regionen Stellungnahmen abgaben, ${ }^{17}$ war die politische Bedeutung des Themas offenkundig. Im Dezember 1993 beschloss die Generalversammlung die Einsetzung einer allen Mitgliedstaaten offen stehenden Arbeitsgruppe, der „Open-ended Working Group to consider all aspects of the question of increase in the membership of the Security Council, and other matters related to the Security Council." ${ }^{18}$

Die Hauptanliegen der reformwilligen Staaten waren eine Verbesserung des repräsentativen Charakters und damit der politischen Legitimität des Sicherheitsrates durch eine Erhöhung der Zahl der nichtständigen und - von weniger Staaten unterstützt - der ständigen Mitglieder sowie eine Verbesserung der Beschlussfähigkeit des Rates durch eine Abschaffung oder Einschränkung des Vetorechts der ständigen Mitglieder (Artikel 27 III der UNCharta). Verwiesen wurde insbesondere auf die stark angewachsene Mitgliedschaft der UN. Führte das erste UN-Jahrbuch (mit Stand vom Juli 1947) 55 Mitgliedstaaten auf, waren es 1963 (zur Zeit der Erhöhung der Zahl der nichtständigen Sitze von ursprünglich sechs auf zehn) bereits 113, während den UN am 1. Januar 1995185 Staaten angehörten (seit 2011

12 UNO, Generalversammlung 1991, S. 1-41.

13 Vgl. Fassbender 1998a, S. 221 f. Texte der Resolutionsentwürfe: Fassbender 2020, S. 88 ff.

14 UNO, Generalversammlung 1992a, S. 11-91.

15 Vgl. Fassbender 1998a, S. $223 \mathrm{f}$.

16 UNO, Generalversammlung 1992 b.

17 Zusammenfassung der Stellungnahmen bei Fassbender 1998a, S. 234 ff.; die Stellungnahmen selbst in Fassbender 2020, Abschnitte 3.2, 3.3 und 3.4.

18 UNO, Generalversammlung 1993. 
sind es 193 Mitgliedstaaten). Das Verhältnis der Gesamtzahl der Mitglieder zur Zahl der Sitze im Sicherheitsrat betrug 19455 zu 1, im Jahr 1963 (vor der Vergrößerung des Rates) 10.3 zu 1 und 199312.3 zu 1. Außerdem wurde eine zu geringe Vertretung der afrikanischen und asiatischen Staaten im Sicherheitsrat und umgekehrt eine Überrepräsentation der westlichen Staaten als Reformgrund angeführt. Hinsichtlich der ständigen Mitglieder wurde eine Verschiebung der globalen Machtverhältnisse geltend gemacht, welche die ständigen Sitze Frankreichs und Grossbritanniens fragwürdig erscheinen lasse und stattdessen eine permanente Vertretung von Ländern wie Japan, Indien, Brasilien oder Deutschland nahelege. Die afrikanischen Staaten forderten vehement eine Vertretung ihres Kontinents in der Kategorie der ständigen Ratsmitglieder. Das Vetorecht der ständigen Mitglieder wurde nicht nur als Hindernis für eine rasche und effektive Reaktion des Sicherheitsrates auf Bedrohungen oder Verletzungen des Weltfriedens kritisiert, sondern auch als anachronistisch, undemokratisch und diskriminierend.

Während die Reformdiskussion in den ersten Jahren noch von einer breiten Übereinstimmung darüber getragen wurde, es sei die Zeit dafür reif, den Sicherheitsrat in seiner Zusammensetzung, seinem Entscheidungsverfahren und seinem Verhältnis zu den anderen Hauptorganen, insbesondere der Generalversammlung, den geänderten Umständen anzupassen, wurden zugleich auch Konfliktlinien offensichtlich - Interessen und Forderungen einzelner Staaten und Staatengruppen, die nicht miteinander vereinbar waren. Die Entwicklungsländer, die in der Generalversammlung die überwältigende Mehrheit bilden (der „Group of 77“ gehören heute 134 der insgesamt 193 Mitgliedstaaten an) und deren Zustimmung wegen des Erfordernisses einer Zweidrittelmehrheit der Mitgliedstaaten für jede Änderung der UNCharta (Artikel 108 und 109) unverzichtbar ist, forderten eine erheblich stärkere Vertretung sowohl in der Gruppe der nichtständigen als auch der ständigen Mitglieder. Andererseits konnte sich der global South nicht auf gemeinsame Kandidaten für neue ständige Sitze einigen, wofür hauptsächlich regionale Rivalitäten verantwortlich waren (Ablehnung eines ständigen Sitzes für Brasilien durch die spanischsprachigen Länder Lateinamerikas, insbesondere Argentinien und Mexiko; Ablehnung eines Sitzes für Indien durch Pakistan; konkurrierende Ansprüche Nigerias, Südafrikas und Ägyptens in Afrika). Die Forderung nach einer wesentlichen Erhöhung der Zahl der nichtständigen Sitze zugunsten der Entwicklungsländer (um etwa zehn Sitze) wurde besonders von westlichen Regierungen zurückgewiesen, die in einem zu großen Sicherheitsrat eine Belastung seiner Arbeitsfähigkeit sahen. Mehrfach nannte die US-Regierung als Obergrenze eines erweiterten Rates 21 Sitze. Die Bemühungen Japans und Deutschlands um einen ständigen Sitz (vorangetrieben in der mit Indien und Brasilien gebildeten „Gruppe der Vier“ oder „G 4“) fanden außerhalb der westlichen Staatengruppe nur wenig Unterstützung; aus der Sicht des Südens würden solche Sitze die ungerechte Dominanz des Nordens im Sicherheitsrat noch verstärken. Energisch abgelehnt wurde die Politik der „G 4“ und insbesondere die deutsche Position von Italien, dem es gelang, nicht wenige Staaten auf seine Seite zu ziehen - hauptsächlich diejenigen „mittelmächtigen“ Länder, die sich selbst keine Hoffnungen auf einen ständigen Sitz machen können, andererseits aber auch nicht zusammen mit den vielen Kleinen und Ganzkleinen in den hinteren Bänken sitzen wollen. Italien lehnte neue ständige Sitze überhaupt ab und warb stattdessen für eine neue Kategorie von rotierenden Sitzen.

Was das von der Mehrheit der UN-Mitgliedstaaten als heute unbegründetes, ja schädliches Privileg abgelehnte Vetorecht der ständigen Mitglieder betrifft, so stellte sich in den 
Beratungen der Open-ended Working Group bald heraus, dass die „Ständigen Fünf“ (P 5) weder seiner Abschaffung noch seiner förmlichen Beschränkung zustimmen würden, womit die Frage praktisch erledigt war, denn für das Inkrafttreten der Charta-Änderung ist bekanntlich neben der genannten Zweidrittelmehrheit der UN-Mitgliedstaaten (d.h. gegenwärtig 129 der 193 Mitglieder) auch die Zustimmung jedes einzelnen ständigen Mitglieds des Sicherheitsrates erforderlich (Artikel 108 und 109 der UN-Charta). Zugleich wurde erkennbar, dass die „P 5“ auch neue ständige Sitze mit einem Vetorecht ablehnten, wie sie von den afrikanischen Staaten und von Deutschland und Japan gefordert wurden.

\subsection{Das Razali Reform Paper von 1997}

Ihren Höhepunkt und Abschluss fand die erste, im Vergleich mit den folgenden produktivste Periode der Reformdiskussion im Frühjahr 1997, als der Präsident der UN-Generalversammlung und Vorsitzende der Open-ended Working Group, Botschafter Razali Ismail (Malaysia), einen Vorschlag in Form des Entwurfs einer Resolution der Generalversammlung präsentierte. ${ }^{19}$ Botschafter Razali regte an, die Generalversammlung solle in einem ersten Schritt mit einer einfachen Mehrheit eine Resolution annehmen, derzufolge der Sicherheitsrat um fünf ständige und vier nichtständige Mitglieder erweitert werden solle. Zwei der neuen ständigen Sitze sollten den ,industrialisierten Staaten“ zustehen und je ein Sitz den Entwicklungsländern in Afrika, Asien sowie Lateinamerika und der Karibik. Die neuen ständigen Mitglieder würden kein Vetorecht besitzen. Je einer der vier neuen nichtständigen Sitze würde Afrika, Asien, Osteuropa sowie Lateinamerika und der Karibik zustehen. Erst in einem zweiten Schritt, so das „Razali Reform Paper“, würden mit einer Zweidrittelmehrheit der Generalversammlung bestimmte Staaten als neue ständige Mitglieder benannt. Eine Woche später würde die Generalversammlung in einem dritten Schritt, ebenfalls mit Zweidrittelmehrheit, die notwendigen Änderungen der UN-Charta gemäß Artikel 108 der Charta beschließen. Zehn Jahre nach dem Inkrafttreten der Änderungen sollte eine Konferenz der Mitgliedstaaten gemäß Artikel 109 der Charta einberufen werden, um die Folgen der Änderungen zu überprüfen.

Diesem Versuch Razalis, die Grundsatzfrage einer Erweiterung des Sicherheitsrates um neue Sitze von der Frage zu trennen, welche Staaten diese Sitze einnehmen würden, und für die Entscheidung der ersten Frage das Hindernis der Zweidrittelmehrheit in der Generalversammlung zu umgehen, war jedoch kein Erfolg beschieden. Razali musste darauf verzichten, seinen Entwurf zur Abstimmung zu stellen, und im November 1998 beschloss die Generalversammlung unter Verweis auf Kapitel XVIII der UN-Charta, sie werde keine Resolution oder Entscheidung in der Frage der Sicherheitsratsreform annehmen ,without the affirmative vote of at least two thirds of the Members of the General Assembly“. ${ }^{20}$ An diesem Beschluss (einem Erfolg einerseits der Gegner neuer ständiger Sitze, andererseits der in der „Group of 77“ und der Blockfreienbewegung zusammengeschlossenen Entwicklungsländer) hat die Generalversammlung bis heute festgehalten.

19 Text des Vorschlags in Fassbender 2020, S. $152 \mathrm{ff}$.

20 UNO, Generalversammlung 1998. Auch in: Fassbender 2020, S. 509. 


\subsection{Der Bericht „A more secure world“ (2004) und die drei Resolutionsentwürfe vom Juli 2005}

Aus der folgenden Periode der Reformdiskussion, die vom Scheitern der Razali-Initiative bis zur Verschiebung des Verhandlungsforums von der Open-ended Working Group zu den Intergovernmental Negotiations im Jahr 2009 datiert werden kann, seien noch der Bericht „A more secure world“ des von Generalsekretär Kofi Annan berufenen High-level Panel on Threats, Challenges and Change vom Dezember $2004^{21}$ sowie die drei Resolutionsentwürfe vom Juli 2005 hervorgehoben.

Vor dem Hintergrund der unvereinbaren Positionen der Anhänger und der Gegner neuer ständiger Sitze stellte der Bericht des High-level Panel zwei Modelle einer Erweiterung des Sicherheitsrates nebeneinander, beide mit einer Gesamtzahl von 24 Mitgliedern. „Modell A“ sah sechs zusätzliche ständige Mitglieder ohne Vetorecht und drei neue nichtständige Mitglieder vor. „Modell B“ schlug dagegen die Schaffung einer neuen Kategorie von Ratsmitgliedern vor, die aus acht Sitzen mit einer (unmittelbar erneuerbaren) Amtszeit von vier Jahren bestehen sollte. Außerdem sollte der Sicherheitsrat um einen (traditionellen) nichtständigen Sitz erweitert werden. ${ }^{22}$ In seinem Bericht „In larger freedom“ von 2005 vermied es der Generalsekretär, eine Präferenz für eines der beiden Modelle zum Ausdruck zu bringen. ${ }^{23}$

Die drei Resolutionsentwürfe vom Juli 2005 bildeten die unterschiedlichen Ansichten der Mitgliedstaaten zwar nicht erschöpfend ab, markierten aber doch die Positionen der um eine Ratsreform aktiv bemühten Staatengruppen. Zu jener Zeit gelangten die deutsche und die japanische Regierung zu der Einschätzung, es sei notwendig, die UN-Mitgliedstaaten zu Entscheidungen über die Ratsreform zu veranlassen, wenn man nicht riskieren wollte, dass der ganze Reformprozess zu einem Stillstand kommen würde. Man hoffte auch, für den eigenen Vorschlag eine so deutliche Mehrheit zu gewinnen, dass die Staats- und Regierungschefs ihm im September 2005 zustimmen würden, wenn sie zur Feier des 60jährigen Bestehens der UN in New York zusammenkämen.

Zunächst brachte die „G 4“ (Brasilien, Deutschland, Indien und Japan) zusammen mit 23 anderen Staaten einen Entwurf ein, der sechs neue ständige und vier neue nichtständige Sitze vorsah. ${ }^{24}$ Je zwei der ständigen Sitze sollten von afrikanischen und asiatischen Staaten besetzt werden, und je einer von einem lateinamerikanischen und einem Staat der WEOGGruppe (Western European and Other States). Offen war hier also nur die Besetzung der beiden Afrika zugedachten Sitze. Von den vier neuen nichtständigen Sitzen sollte je einer Afrika, Asien, Osteuropa sowie Lateinamerika und der Karibik zustehen. Die Unterstützung des Entwurfs blieb deutlich hinter den Erwartungen der G 4 zurück. Insbesondere gelang es nicht, die Länder der afrikanischen Staatengruppe als „,co-sponsors“ zu gewinnen.

Die afrikanischen Staaten brachten stattdessen einen eigenen Resolutionsentwurf ein. ${ }^{25}$ Nach diesem sollte die Zahl der Sicherheitsratssitze um insgesamt elf erhöht werden, und zwar um sechs ständige Sitze (je zwei für Afrika und Asien, je einen für Lateinamerika

21 UNO, Generalsekretär 2004.

22 Vgl. auch Rittberger/Baumgärtner 2005, S. 309 ff.

23 Vgl. UNO, Generalsekretär 2005, Abs. 168 ff. Analyse bei Fassbender 2006.

24 UN-Dokument A/59/L.64 v. 06.07.2005, in: Fassbender 2020, S. 660 ff.

25 UN-Dokument A/59/L.67 v. 14.07.2005, in: Fassbender 2020, S. 750 ff. 
und die Karibik sowie für WEOG) und fünf nichtständige (zwei für Afrika und je einen für Asien, Osteuropa sowie Lateinamerika und die Karibik). Der wichtigste Unterschied zum G 4-Entwurf betraf das Vetorecht. Die afrikanischen Staaten fordern nämlich ausdrücklich, dass die neuen ständigen Mitglieder ,dieselben Vorrechte und Privilegien wie die gegenwärtigen ständigen Mitglieder“ genießen sollen, ,einschließlich des Vetorechts“. Diese Forderung stand im Einklang mit einem Beschluss des Exekutivrates der Afrikanischen Union vom März 2005, dem sogenannten „Ezulwini Consensus“, in dem es hieß, dass Afrika zwar im Prinzip das Vetorecht ablehne, aber der Auffassung sei, dass dieses, so lange es bestehe, aus Gründen der Gerechtigkeit und Gleichberechtigung allen ständigen Mitgliedern des Sicherheitsrates zustehen solle. ${ }^{26}$

Der dritte Entwurf stammte von Italien, Mexiko, Pakistan und neun weiteren Staaten (darunter Argentinien, Kanada, Spanien und die Türkei) - der „Uniting for Consensus“ (UfC)-Gruppe. ${ }^{27}$ Er sah vor, die Zahl der Mitglieder des Sicherheitsrates auf insgesamt 25 zu erhöhen, und zwar allein durch eine Hinzunahme von zehn neuen nichtständigen Mitgliedern. Die Gruppe warb mit dem Argument, ihr Entwurf erhöhe die Chancen aller Mitgliedstaaten, einen Platz im Sicherheitsrat einzunehmen, und entspreche dem Prinzip der souveränen Gleichheit der Mitglieder. Die nichtständigen Mitglieder sollten wie bisher von der Generalversammlung gewählt werden; auch die in Artikel 23 Abs. 1 der UN-Charta genannten Kriterien (nämlich der Beitrag von Mitgliedstaaten zur Wahrung des Weltfriedens und der internationalen Sicherheit und zur Verwirklichung der sonstigen Ziele der Organisation sowie eine ausgewogene geographische Verteilung der Sitze) wurden übernommen. Wegfallen sollte hingegen nach dem Entwurf das bisherige Verbot einer unmittelbaren Wiederwahl nichtständiger Mitglieder. Es sollte den einzelnen Regionalgruppen überlassen bleiben $\mathrm{zu}$ entscheiden, ob ein ausscheidendes Mitglied wiedergewählt wird oder in welcher Weise es zu einer rotierenden Mitgliedschaft im Sicherheitsrat kommt. Der Entwurf ordnete die zwanzig nichtständigen Sitze den Regionen wie folgt zu: sechs Sitze sollten von den afrikanischen Staaten besetzt werden, fünf von den asiatischen Staaten, vier von den Staaten Lateinamerikas und der Karibik, drei von den WEOG-Staaten sowie zwei von den osteuropäischen Staaten. Italien und seine Mitstreiter modifizierten mit diesem Entwurf ihre früheren Vorschläge, die eine neue dritte Kategorie von Mitgliedern des Sicherheitsrates - gleichsam zwischen den heutigen ständigen und den nichtständigen Mitgliedern stehend - und für diese ein detailliertes Rotationsschema vorsahen.

Jedoch wurden die drei Resolutionsentwürfe nicht zur Abstimmung gestellt, da keiner von ihnen mit der erforderlichen Zweidrittelmehrheit rechnen konnte. Zudem lehnten die USA und China eine Abstimmung ausdrücklich ab. In der 60. Sitzungsperiode der Generalversammlung wurden die Entwürfe der G 4 und der afrikanischen Staaten erneut eingebracht, der erste allerdings nur noch von Brasilien, Deutschland und Indien. ${ }^{28}$ Japan verhandelte informell mit den USA und China über ein Modell, nachdem der Sicherheitsrat um sechs Sitze erweitert werden würde. Zwei neue Sitze sollten an asiatische Staaten gehen, zwei an Afrika und je einer an Lateinamerika und WEOG. Für diese Sitze kandidierende

26 African Union, Executive Council, 7th Extraordinary Session, 07./08.3.2005, Dokument Ext/EX.CL/2(VII), Teil C.e), Abs. 3, in: Fassbender 2020, S. 745 ff.

27 UN-Dokument A/59/L.68 v. 21.07.2005, in: Fassbender 2020, S. 712 ff., 796 f.

28 Vgl. UN-Dokumente A/60/L.46 v. 05.01.2006, in: Fassbender 2020, S. 672, und A/60/L. 41 v. 14.12.2005. 
Länder sollten, sofern sie in der Generalversammlung eine Zweidrittelmehrheit erreichten, einen ständigen Sitz erhalten, die übrigen einen nichtständigen mit der Möglichkeit der unmittelbaren Wiederwahl. Mit diesem Vorschlag suchte die japanische Regierung besonders den USA entgegenzukommen, die nur eine kleine Zahl von zusätzlichen Sitzen zu unterstützen bereit waren. Japan brachte aber keinen offiziellen Entwurf ein. Auch die „Uniting for Consensus“-Gruppe verzichtete darauf, der Generalversammlung ihren Resolutionsentwurf vom Vorjahr erneut vorzulegen. Wie schon in der 59. Sitzungsperiode kam es auch in der sechzigsten nicht zu einer Abstimmung über die Entwürfe.

\subsection{Der L.69-Resolutionsentwurf vom September 2007}

Im September 2007 legte eine Gruppe von 25 Mitgliedstaaten aus Afrika, Lateinamerika und der Karibik, Asien und dem Pazifik (darunter Brasilien, Indien, Nigeria und Südafrika) einen Resolutionsentwurf vor (UN-Dokument A/61/L.69), welcher dieser Staatengruppe auch den Namen „L.69 Group“ gab..$^{29}$ Der Entwurf, der nicht zur Abstimmung in der Generalversammlung gestellt wurde, enthielt insbesondere die an den gewählten Präsidenten der 62 . Sitzungsperiode der Generalversammlung gerichtete Aufforderung ,to take immediate steps to facilitate results-oriented intergovernmental negotiations". Er sprach sich ferner für eine Erweiterung des Sicherheitsrates um neue ständige und nicht-ständige Mitglieder und eine stärkere Vertretung der Entwicklungsländer, einschließlich der Inselstaaten und der kleinen Staaten, aus. Im Rat vertreten sein sollten „reflective of contemporary world realities“ auch entwickelte Staaten und solche mit einer Übergangswirtschaft (transition economy).

Im Jahr 2012 präsentierte die L.69-Gruppe, die heute etwa vierzig Mitgliedstaaten zählt, einen weiteren Resolutionsentwurf ${ }^{30}$, der die afrikanischen Staaten gewinnen wollte, indem für neue ständige Mitglieder des Sicherheitsrates das Vetorecht vorgesehen wurde: „[T]he new permanent members shall have the same prerogatives and privileges of those of the current permanent members, including the right of veto“. Die Zahl der nichtständigen Sitze sollte auf sechzehn erhöht werden. Zwei der neuen Sitze waren für die afrikanischen Staaten bestimmt und je ein Sitz für Asien, Osteuropa, Lateinamerika und die Karibik, die WEOG-Staaten sowie für „Small Island Developing States across all regions“. Ferner sah der Entwurf zwei neue ständige Sitze für Afrika vor, zwei für Asien, einen für Lateinamerika und die Karibik und einen für WEOG. Entsprechend sollte der reformierte Sicherheitsrat im Ganzen 27 Mitglieder haben. Auch dieser Resolutionsentwurf gelangte nicht zur Abstimmung.

\subsection{Der Übergang zu den Intergovernmental Negotiations (IGN)}

Im September 2007 beschlossen die UN-Mitgliedstaaten, die Reformdiskussion in einem neuen Format, den Intergovernmental Negotiations (IGN) in informellen Besprechungen des Plenums der Generalversammlung, fortzuführen. ${ }^{31}$ Die Open-ended Working Group, die ebenfalls allen Mitgliedstaaten zur Mitarbeit offen gestanden hatte, wurde allerdings nicht förmlich aufgelöst, sodass die dortige Arbeit jederzeit wieder aufgenommen werden könnte.

29 Text: Fassbender 2020, S. $674 \mathrm{f}$.

30 Text: Fassbender 2020, S. $998 \mathrm{f}$.

31 Entscheidung 61/561 der UN-Generalversammlung v. 17.09.2007, in: Fassbender 2020, S. 630. 
Der Hauptgrund für diese Änderung lag in der Enttäuschung vieler Staaten, insbesondere der G 4, über den schleppenden Verlauf der Reformdiskussion in der Arbeitsgruppe, die seit ihrer Errichtung ihre Beschlüsse ,by general agreement“ gefasst hatte und sich daher nur auf sehr allgemeine Formulierungen hatte einigen können. Mit dem IGN-Format verband sich unausgesprochen die Erwartung einer Möglichkeit von Abstimmungen über Resolutionsentwürfe, so wie sie in den Hauptausschüssen der Generalversammlung besteht, und eines rascheren Übergangs zu Abstimmungen im Plenum der Generalversammlung. Die Befürworter der IGN versprachen sich auf diesem Weg „echte“ Verhandlungen über konkrete Vorschläge (,text-based negotiations“) anstelle der Aneinanderreihung von Stellungnahmen in der Arbeitsgruppe. Allerdings verstrich ein volles Jahr, bis man sich auf die Modalitäten der IGN verständigen konnte, ${ }^{32}$ und auch dies nur mit Kompromissen, die unterschiedliche Auslegungen erlauben. ${ }^{33}$ Nachdem die Verhandlungen am Anfang des Jahres 2009 begonnen hatten, verging ein weiteres Jahr bis zur Vorlage eines ersten ,negotiation text“ durch den Vorsitzenden der IGN, Botschafter Zahir Tanin (Afghanistan) am 10. Mai 2010 ${ }^{34}$. Bei diesem 29 Seiten langen Dokument handelt es sich um aneinander gereihte Vorschläge der Mitgliedstaaten zu einzelnen Reformthemen (categories of membership, the question of the veto, regional representation, size of an enlarged Council and working methods, relationship between the Council and the General Assembly), die im copy \& paste-Verfahren umfassenderen Stellungnahmen der betreffenden Staaten entnommen wurden. Auf diese Weise sollten die unterschiedlichen Positionen deutlicher zum Ausdruck kommen und eine Annäherung erleichtert werden. Außerdem sollten sich die Verhandlungen von nun an auf einen einzigen Text konzentrieren. Allerdings erhoben sofort mehrere Staaten Einspruch gegen eine nach ihrer Ansicht verkürzte oder missverständliche Wiedergabe ihrer Auffassungen. Der „negotiation text" wurde in der Folge mehrfach revidiert und durch "framework documents", „elements of convergence“ und „elements of commonality“ ersetzt oder ergänzt. ${ }^{35}$ Diese unterscheiden sich unter anderem durch das Vorhandensein oder Fehlen einer namentlichen Zuschreibung (attribution) einer Position oder eines Vorschlags zu einem bestimmten Staat.

Im Rückblick lässt sich feststellen, dass die Ersetzung der Open-ended Working Group durch die IGN nicht zu dem von den Befürwortern substantieller Verhandlungen erhofften Erfolg geführt hat. Vergleicht man die im Jahr 2008 unmittelbar vor Beginn der IGN vertretenen Positionen mit dem heutigen Diskussionsstand, konnte eine wirkliche Annäherung in den Hauptstreitpunkten nicht erreicht werden. Die G 4 bezeichnen den IGN-Prozess heute sogar als ,a convenient smokescreen to hide behind for those who do not wish to see any reform in the Security Council““36. Eine Mehrheit der Mitgliedstaaten, insbesondere kleinere Staaten und die Entwicklungsländer, scheint heute den Status quo einer Reform vorzuziehen, welche neue ständige oder semiständige Sitze einführte. Vor diesem Hintergrund erscheinen Berichte glaubhaft, denen zufolge sich an den IGN nur etwa ein Viertel der Mitglieder der UN aktiv beteiligt hat. Die Enttäuschung vieler Mitgliedstaaten über den geringen Fortschritt, der in den IGN erzielt werden konnte, kommt auch in einer Äußerung des

32 Entscheidung 62/557 der UN-Generalversammlung v. 15.09.2008, in: Fassbender 2020, S. $640 \mathrm{ff}$.

33 Vgl. Swart 2013, S. 23.

34 Text in: Fassbender 2020, S. $874 \mathrm{ff}$.

35 Vgl. für die Einzelheiten Fassbender 2020, S. $27 \mathrm{ff}$.

36 Vgl. Abs. 10 der Stellungnahme Indiens im Namen der G 4 v. 31.08.2020 zum Entwurf A/74/L.88 der Entscheidung der Generalversammlung; Anlage zu UNO, Generalversammlung $2020 \mathrm{f}$. 
Präsidenten der Generalversammlung in der 74. Sitzungsperiode, Botschafter Tijjani Muhammad-Bande (Nigeria), am Beginn der (aufgrund der Covid-Pandemie kurz darauf abgebrochenen) IGN im Februar 2020 zum Ausdruck:

In my view, the most appropriate platform to pursue this reform is still within the framework of the IGN. However, we put this in danger, if we fail to make progress, we will be thereby entrenching the cynicism that has led some delegations to voice frustration, to the extent of questioning the value of their continued participation. Accordingly, I ask all delegations to utilize the opportunity that this IGN session provides to engage constructively. We must give this process a chance. ${ }^{37}$

Mit einer geringfügigen Änderung der bisher benutzten Formulierungen definierte der Präsident der Generalversammlung die vier „key issues“ der IGN in der 74. Sitzungsperiode so:

i. Size of an enlarged Council, as well as the issue of regional representation;

ii. Abolition of, or extension of veto power, and its use;

iii. Expansion or non-expansion in both permanent and non-permanent categories of membership;

iv. Support for, or opposition to, Text-Based Negotiation (TBN) and Attribution and the emerging debate about the process. ${ }^{38}$

\section{Der gegenwärtige Stand der Reformdebatte}

Die letzte Entscheidung der UN-Generalversammlung zur Frage der Reform des Sicherheitsrates (74/569) datiert vom 31. August 2020. Zum weiteren Verfahren der Intergovernmental Negotiations beschloss die Generalversammlung schriftlich

to immediately continue intergovernmental negotiations on Security Council reform in informal plenary of the General Assembly at its seventy-fifth session, [...], building on the informal meetings held during its seventy-fourth session, as reflected in the letter dated 29 July 2020 from the Co-Chairs ${ }^{[39]}$, and on the revised elements of commonality and issues for further consideration, circulated on 7 June 2019, as well as the positions of and proposals made by Member States, reflected in the text and its annex circulated on 31 July 2015, to help inform its future work. ${ }^{40}$

Die Entscheidung wiederholte, von den neuen Daten abgesehen, die des Vorjahres vom 25. Juni $2019^{41}$. Grundlage der künftigen Besprechungen bleiben damit das „Framework Document" und sein Anhang vom 31. Juli $2015^{42}$ (mit einer attribution oder Zuschreibung der Positionen zu bestimmten Staaten) sowie die „Revised elements of commonality and issues

37 UNO, Generalversammlung 2020b.

38 UNO, Generalversammlung 2020b.

39 Vgl. UNO, Generalversammlung 2020e.

40 UNO, Generalversammlung $2020 \mathrm{f}$.

41 Entscheidung 73/554 der UN-Generalversammlung v. 25.06.2019, in: Fassbender 2020, S. 985 f., sowie in UNO, Generalversammlung 2019a, S. 2.

42 Text in: Fassbender 2020, S. $943 \mathrm{ff}$. 
for further consideration" vom 7. Juni $2019^{43}$ (ohne eine solche Zuschreibung). Besonders die afrikanischen Staaten legen Wert auf die weitere Berücksichtigung des Dokuments von 2015 als ,the main reference document for the intergovernmental negotiations “444, während die Uniting for Consensus-Gruppe dem Dokument mit Vorbehalten begegnet ${ }^{45}$. Ein neuer Text, vergleichbar denen von 2015 und 2019, konnte in den Pandemie-bedingt abgebrochenen IGN-Treffen der 74. Sitzungsperiode ${ }^{46}$ nicht vereinbart werden.

Wenn insbesondere seitens der G 4-Staaten die Aufnahme von ,text-based negotiations“" gefordert wird (so der deutsche Ständige Vertreter in der Generalversammlung am 25. November 2019: „To this day, we have not succeeded in getting closer to text-based negotiations ${ }^{647}$ ), ist damit gemeint, von den Zusammenstellungen der unterschiedlichen Positionen durch die IGN-Vorsitzenden zu Verhandlungen (und Abstimmungen) über konkrete Reformvorschläge überzugehen, welche in einen Entwurf einer Resolution der Generalversammlung münden würden (,,we need a concise negotiating text that will allow us to finally start concrete, results-oriented negotiations ${ }^{\text {“48 }}$ ). Dagegen betonen die Uniting for Consensus-, aber auch die arabischen Staaten, es müssten weiterhin „consensus-based solutions ${ }^{\star 49}$ gesucht werden: „Only through a transparent process that takes into account the voices of all Member States will we be jointly able to define a consensual path to reform ${ }^{\text {"50 }}$. Auch Russland und China sprechen sich in diesem Sinne aus. So erklärte der russische Ständige Vertreter: „We cannot make progress in reforming the Security Council by imposing on Member States facilitators' texts, negotiation documents or any other initiatives that have not been agreed to by all the participants in the process ${ }^{651}$, während der chinesische Vertreter warnte vor einem ,rushing into text-based negotiations, setting artificial timelines or even trying to force through premature reform proposals ${ }^{\text {“52 }}$. Neuerdings verlangen die G 4 auch die Anwendung der Geschäftsordnung der Generalversammlung (mit den Regeln über Abstimmungen im Abschnitt XII) auf die IGN, was von der Uniting for Consensus-Gruppe und anderen Staaten mit Verweis auf den informellen Charakter der IGN-Diskussionen abgelehnt wird. ${ }^{53}$

Zum Thema der Erweiterung des Sicherheitsrates erklärt das Papier vom 7. Juni 2019 im Abschnitt II („Commonalities“) Unterabschnitt 3 („Size of an enlarged Council and Working Methods of the Council'"):

43 Text in: Fassbender 2020, S. $980 \mathrm{ff}$.

44 Botschafter Kabba (Sierra Leone) in: UNO, Generalversammlung 2019b, S. 3. Im gleichen Sinne Botschafterin King (Saint Vincent and the Grenadines) für die L.69-Gruppe, ebd., S. $8 \mathrm{f}$.

45 Vgl. die Stellungnahme Italiens im Namen der UfC v. 31.08.2020 zum Entwurf A/74/L.88 der Entscheidung der Generalversammlung; Anlage zu UNO, Generalversammlung $2020 \mathrm{f}$.

46 Vgl. oben Text bei Fn. 4.

47 Botschafter Heusgen in: UNO, Generalversammlung 2019b, S. 1.

48 UNO, Generalversammlung 2019b, S. 2.

49 Vgl. Botschafter Alotaibi (Kuwait) für die Gruppe der arabischen Staaten in: UNO, Generalversammlung 2019b, S. 5.

50 Botschafterin Zappia (Italien) für die UfC-Gruppe in: UNO, Generalversammlung 2019b, S. 7.

51 Botschafter Polyanskiy in: UNO, Generalversammlung 2019b, S. 17.

52 Botschafter Zhang Jun in: UNO, Generalversammlung 2019b, S. 18.

53 Vgl. Abs. 4 und 9 der Stellungnahme Indiens im Namen der G 4 v. 31.08.2020 zum Entwurf A/74/L.88 der Entscheidung der Generalversammlung; Anlage zu UNO, Generalversammlung $2020 \mathrm{f}$. 
An enlarged Security Council should consist of a total of members in the mid-20s, within an overall range of 21-27 seats, with the exact number to emerge from the discussion of Member States on the key issues of categories of membership and regional representation, and with the total size ensuring a balance between the representativeness and effectiveness of an enlarged Council. ${ }^{54}$

Im folgenden Unterabschnitt 4 heißt es zur Frage der „categories of membership“:

In an enlarged Security Council, the expansion of the category of 2-year term non-permanent members is accepted by all Member States as part of a comprehensive Security Council reform. The Council's expansion in other categories (permanent, longer term seats, transitional options) remains to be agreed through negotiations by Member States. ${ }^{55}$

Zur Frage der „regional representation“ wird im Unterabschnitt 6 als gemeinsamer Standpunkt formuliert, die Vertretung von unterrepräsentierten und nicht repräsentierten Regionen und Gruppen solle verbessert werden, ohne die Arbeitsfähigkeit des Sicherheitsrates zu beeinträchtigen. Besondere Aufmerksamkeit solle einer gerechten Vertretung (equitable representation) der Entwicklungsländer, der kleinen Staaten, der Small Island Development States, der arabischen Staaten und Afrikas geschenkt werden. ${ }^{56}$

Hier aber enden die „commonalities“ der Mitgliedstaaten. Der folgende Abschnitt III der „Revised elements“ listet unter der Überschrift „Issues for further consideration“ die meisten der offenen Fragen auf, darunter die Kriterien für die Wahl der nichtständigen Mitglieder, die Zahl der nichtständigen Mitglieder im Verhältnis zur Zahl der UN-Mitgliedstaaten sowie zur Zahl der Mitglieder der Regionalgruppen, und die Rolle der Regionalgruppen im Verfahren der Wahl der Mitglieder des Rates. Über die Kategorien der Mitglieder eines reformierten Sicherheitsrates heißt es (III.4.a):

Member States express their preference for one or more of the following options, in a reformed Security Council:

- Enlargement of the Security Council in both the permanent and 2-year non-permanent categories;

- Enlargement of the Security Council with 2-year term non-permanent members and creation of longer-term non-permanent seats with the possibility of immediate reelection;

- Enlargement of the Security Council with 2-year term non-permanent seats. ${ }^{57}$

Eben diese Alternativen wurden allerdings schon zu Beginn der Reformdebatte kontrovers debattiert. Wie zuvor beschränkt sich die Einigkeit auf eine Erweiterung des Rates um eine gewisse Zahl von nichtständigen Mitgliedern in der bisherigen Form (zweijährige Amtszeit ohne Möglichkeit der unmittelbaren Wiederwahl), während nur ein Teil der Mitgliedstaaten

54 Fassbender 2020, S. 981.

55 Fassbender 2020, S. 981.

56 Vgl. Fassbender 2020, S. 982, sowie die Zusammenfassung der IGN-Diskussionen v. 04./05.03.2020 in UNO, Generalversammlung 2020e.

57 Fassbender 2020, S. $982 \mathrm{f}$. 
zusätzliche ständige Sitze (mit oder ohne Vetorecht) oder zusätzliche nichtständige Sitze mit einer längeren Amtszeit und/oder der Möglichkeit einer unmittelbaren Wiederwahl unterstützt. Die afrikanischen Staaten halten die beiden bestehenden Kategorien von Mitgliedern für ausreichend: ,,a Security Council with an asymmetrical category of permanent members“ (d.h. ständigen Mitgliedern mit einem unterschiedlichen Status) ,would be completely unbalanced, while a category of non-permanent members with ambiguous mandates and subdivisions would complexify the Council and render it ineffective. ${ }^{\text {"58 }}$

Auch die Ausführungen des Papiers von 2019 zum Veto-Recht (III.5.b) zeigen kaum einen Fortschritt und lassen leicht die jeweiligen Befürworter erkennen. Neu ist im Vergleich zur Diskussion in den 1990er Jahren ${ }^{59}$ allein der Absatz über einen freiwilligen Verzicht auf die Ausübung des Vetorechts in bestimmten Fällen:

The following options in regard to the veto are being discussed by Member States:

- In the case of enlargement of the Security Council in the permanent category:

- Extension of the veto to all permanent members; or

- No extension of the veto; or

- Extension of the veto to be decided on in the framework of a review.

- Progressive restraint.

- Abolition of the veto.

- Opposition in principle to the veto, with the caveat that as long as it exists, it should be made available to all permanent members of the Security Council including any potential permanent members from Africa - based on the common African position reflected in the Ezulwini Consensus adopted by the African Union in March 2005.

- Voluntarily refrain from the use of the veto in cases of mass atrocity crimes. Initiatives in this regard include the „Political statement on the suspension of the veto in case of mass atrocities " presented by France and Mexico during the 70th session of the GA and the „Code of Conduct regarding Security Council action against genocide, crimes against humanity and war crimes " by the Accountability, Coherence and Transparency (ACT) Group. ${ }^{60}$

58 Botschafter Mouanda (Kongo), in: UNO, Generalversammlung 2019c, S. 16.

59 Hierzu ausführlich Fassbender 1998a, S. 263 ff.

60 Fassbender 2020, S. 983. 


\section{Aktuelle Positionen einzelner Staatengruppen sowie der ständigen Mitglieder des Sicherheitsrates in der 74. Sitzungsperiode der UN-Generalversammlung}

\section{Group of Four (Brazil, Germany, India, Japan)}

"[A]n expansion of the Security Council [SC] in both categories is indispensable to making this body more representative, legitimate and effective. [...] Broader membership of the SC - especially with increased and enhanced representation of Africa - will allow it to preserve its credibility and create the political backing needed for the peaceful resolution of today's international crises. [...] We express our support for adequately reflecting the Common African Position, as contained in the Ezulwini Consensus and the Sirte Declaration.“ (Mr. Heusgen, Germany, A/74/PV.33, p. 2.)

\section{Group of African States (54 members)}

„The Common African Position on the reform of the SC, as articulated in the Ezulwini Consensus and the Sirte Declaration, is widely recognized in the Assembly. [...] Africa demands no less than two permanent seats with all the prerogatives and privileges of permanent membership, including the right of veto, and five non-permanent seats. Even though Africa is opposed in principle to the veto, it is of the view that so long as it exists and as a matter of common justice, it should be made available to all permanent members of the SC. [...] The selection of such representatives will be done by our regional organization, the African Union.“ (Mr. Kabba, Sierra Leone, A/74/PV.33, pp. 3-4.)

\section{Nordic countries (Denmark, Finland, Iceland, Norway, Sweden)}

"[T] he Nordic countries seek a more transparent, accountable and representative SC, better equipped to address current global challenges and that better reflects current global realities, not least in its representation. That requires a balanced expansion of the Council, including the increased representation of developing countries, greater possibilities for small States to serve as elected members and certainly ensuring that Africa takes its rightful place on the Council through an expansion of both permanent and non-permanent seats for Africa, redressing the historical injustice done to the African continent." (Ms. Juul, Norway, A/74/PV.33, p. 4.)

\section{Group of Arab States (22 members)}

„The primary and broader goal of Council reform and expansion is to ensure an equitable and sound regional and geographical representation in the membership of an expanded Council. Against that backdrop, I reaffirm that, due to its political and cultural character, the Arab Group deserves, as a stand-alone group, a broader representation in the expanded SC. [...] [T] he Arab Group believes that fair representation requires proportionate Arab representation in the non-permanent category [...] [and] permanent Arab representation endowed with all the legal powers of a permanent seat". (Mr. Alotaibi, Kuwait, A/74/ PV.33, p. 6.) 


\section{Uniting for Consensus (UFC) group (includes Argentina, Canada, Italy, Mexico, Pakistan, Republic of Korea, Spain and Turkey)}

"The UFC group firmly believes that the SC needs to become truly representative, accountable, democratic, transparent and effective. [...] We propose creating new, longerterm non-permanent seats with the possibility of immediate re-election, and increasing the number of two-year-term non-permanent seats. The longer-term seats would fulfil the legitimate desire of some Member States to make a greater contribution to the work of the Council and at the same time foster a fairer system of rotation. The SC would consist, then, of 26 members, 21 of which would be non-permanent, assigned as follows: six seats to the Group of African States, three of which would be longer-term seats; five seats to the Group of Asia-Pacific States, three of which would be longer-term seats; four seats to the Group of Latin American and Caribbean States, two of which would be longer-term seats; three seats to the Group of Western European and other States, one of which would be a longer-term seat; two seats to the Group of Eastern European States; and one seat reserved for small island developing States and small States. That rotating seat would not prevent States from running within their regional group but would instead be an additional way for them to gain access to the SC.

That reform model would greatly enhance regional representation. Africa would constitute the largest regional group in the reformed Council, the Asia-Pacific region would have the highest percentage increase, the small island developing States and small States would have greater access to the Council and both Latin America and Eastern Europe would double their representation. Our proposed distribution would also allow for an increased and more stable representation of cross-regional groupings, such as the Group of Arab States.“ (Mrs. Zappia, Italy, A/74/PV.33, pp. 31-32.)

\section{Caribbean Community (CARICOM) (15 members)}

„As small, weak and defenceless States, the members of CARICOM are particularly sensitive to the need to strengthen the legitimacy of the Council by ensuring equitable representation and an increase in membership. [...] CARICOM has proposed that a reformed $\mathrm{SC}$ should provide for a rotating seat for small island developing States, which can bring a unique perspective to the Council's deliberations and whose particular vulnerabilities are increasingly recognized.“(Ms. McGuire, Grenada, A/74/PV.33, p. 11.)

\section{France}

„France supports enlarging both of the Council's membership categories. We therefore support the candidacy of Germany, Brazil, India and Japan as new permanent members, as well as the strengthened presence of African countries among the permanent and nonpermanent members. An enlarged Council could therefore have as many as 25 members, including new permanent and non-permanent members, who should be proposed by all members of the General Assembly and not just their regional group, which would be contrary to the principles of the Charter. Such an expansion would make the SC more representative of the world of today and would strengthen its authority while preserving its executive and operational nature. 
With regard to the question of the veto, we know it is a clearly sensitive issue, and it is up to the States seeking the granting of a permanent seat to make up their own minds. The dual goal must still be to first strengthen the SC's legitimacy and then its own capacity to meet its responsibilities in maintaining international peace and security. It is in that spirit that France proposed several years ago that the five permanent members of the Council collectively and voluntarily suspend the use of the veto in cases of mass atrocities. That voluntary approach does not require a review of the Charter of the United Nations but rather a simple political commitment. Today that initiative, which we put forward with Mexico, is supported by 102 countries, and we call on all Member States that have not yet joined it, particularly the other permanent members of the SC, to do so." (Mr. De Rivière, A/74/PV.33, p. 13.)

\section{Russian Federation}

„As a permanent member of the SC, Russia notes how essential it is to make that body more representative and balanced, above all by increasing its representation of developing States in Africa, Asia and Latin America. We are in favour of righting a historical injustice with regard to Africa, whose number of Council seats corresponds neither to its current role in international affairs nor to the number of African States as a whole.

However, the efforts to expand the Council should not have a negative impact on its ability to react quickly and effectively to emerging challenges. In that context, we are in favour of maintaining the SC's compact character. Its optimal size should not exceed the low twenties. We believe that any ideas that would end up infringing on the prerogatives of the current permanent members of the SC, including the institution of the veto, are unacceptable. It is important to remember that the veto is an important factor in encouraging the members of the Council to seek balanced solutions. Its use or threat of use has frequently rescued the United Nations from associating itself with dubious ventures." (Mr. Polyanskiy, A/74/PV.33, pp. 16-17.)

\section{China}

"Today the world is undergoing greater change than we have seen in a century, with the collective rise of developing countries being a defining feature. China supports reasonable and necessary reform of the SC to meet the needs of the times. Priority should be given to increasing the representation and say on the Council of developing countries, especially African countries. Most States Members of the United Nations are small- or medium-sized. However, 63 countries have never yet held a seat on the SC, and some small and medium-sized countries have managed it once in 40 or 50 years. This is regrettable and unfair. Reform must increase the opportunities for small- and medium-sized countries to sit on the Council and participate in its decision-making processes. That is the only way to make it more democratic, transparent and efficient.“ (Mr. Zhang Jun, A/74/PV.33, p. 18.)

\section{United States of America}

„As we have previously stated, the United States remains open, in principle, to a modest expansion of the SC for both permanent and non-permanent members. We believe that that must be pursued in a way that will not diminish the SC's effectiveness or its efficiency and 
that will not alter or expand the use of the veto. We recommend carefully considering the ability and willingness of any potential Council member to contribute to the maintenance of international peace and security. A Member State's capacity to shoulder the considerable responsibility that comes with sitting on the SC should be taken into account.

The SC is an important tool to address today's most pressing threats to international peace and security, but it occasionally falls short. A well-executed expansion of the Council could help modernize the organ to better reflect twenty-first century global realities and increase its effectiveness. We remain firm, however, that any alteration of the current structure must be agreed to by consensus." (Mr. Barkin, A/74/PV.34, p. 12.)

\section{United Kingdom}

„We are strong supporters of reform that would enable the Council to better reflect the realities of the twenty-first century, become more representative and retain its ability to respond quickly and decisively to challenges to international peace and security. To that end, the United Kingdom supports a modest expansion of the SC in both the permanent and non-permanent categories. We support the creation of new permanent seats for India, Germany, Japan and Brazil, as well as permanent African representation on the Council.

We support the expansion of the non-permanent category of membership to enable broader participation from around the globe on a rotating basis. It is vital to ensure that the Council benefits from the perspectives of as wide a range of Member States as possible if we are to address challenges to international peace and security effectively and equitably. Such modest expansion would bring the SC's membership to somewhere in the mid-20s. An expanded SC would have to retain a strong degree of efficiency and nimbleness in tackling what are often complex and fast-moving debates. That means we would need to think carefully about what an expanded SC would mean for working methods.

Where the veto is concerned, we do not believe that disagreement in this area should prevent progress in other areas. For its part, the United Kingdom has not used the veto since 1989, three decades ago. We also support the code of conduct of the Accountability, Coherence and Transparency group regarding SC action against genocide, crimes against humanity and war crimes, and we call on all States, including the other permanent members of the SC, to do likewise." (Mr. Allen, A/74/PV.34, p. 19-20.)

\section{4 (Zwischen-)Bilanz und Perspektiven}

1. Auch fast dreißig Jahre nach dem Beginn der Debatte über eine Reform des Sicherheitsrates konnten die UN-Mitgliedstaaten keine Einigung in den Hauptfragen erreichen. Allerdings ist diese Debatte in der UN-Generalversammlung und den von ihr eingesetzten Foren (Open-ended Working Group, Intergovernmental Negotiations) bis heute auch kontinuierlich fortgesetzt worden, was ein weiterbestehendes Interesse der Mitgliedstaaten an einer Reform zeigt. Ob es neben zusätzlichen nichtständigen Sitzen in der bisherigen Form auch neue ständige Sitze oder eine neue, dritte Kategorie von Sitzen geben soll, ist nach wie vor ebenso umstritten wie die Zahl der Sitze und ihre Zuordnung zu Regionen, Staatengruppen oder einzelnen Staaten. Keine Einigkeit gibt es auch über die - mit der künftigen Struktur des Rates unmittelbar verknüpfte - Frage der Abstimmungsregeln, insbesondere das Veto- 
recht der heutigen ständigen sowie möglicherweise künftiger zusätzlicher Mitglieder. In allen diesen Fragen (die so mit einander verbunden sind, dass die Modifikation einer Antwort sich sogleich auf alle anderen Antworten auswirkt) tritt die Reformdiskussion seit vielen Jahren auf der Stelle.

2. Eine ,principled reform“, d.h. eine Reform, die sich an bestimmten, zuerst vereinbarten Prinzipien oder Grundsätzen orientieren würde, ${ }^{61}$ ist chancenlos geblieben, weil die beteiligten Staaten jeden in Betracht kommenden Grundsatz (wie den repräsentativen Charakter des Sicherheitsrates, oder seine Legitimität, oder die Effektivität seiner Arbeit, oder die Rechtsgleichheit der Staaten, oder die Demokratisierung der Vereinten Nationen) sogleich am Maßstab seiner Wirkung auf die jeweils eigenen nationalen Interessen überprüft haben. Ein bestimmter Grundsatz ist also von einem Staat oder einer Staatengruppe in der Diskussion auf- oder abgewertet worden je nachdem, ob seine Anwendung die Interessen des Staates oder der Staatengruppe zu fördern oder zu beeinträchtigen versprach. Auf diese Weise haben sich die Grundsätze wechselseitig neutralisiert.

3. In ähnlicher Weise konnten sich in der Diskussion zu keinem Zeitpunkt Gemeinschaftswerte der internationalen Gemeinschaft ${ }^{62}$ (wie die Wahrung des Weltfriedens oder die Sicherung der Menschenrechte oder die Erhaltung des ökologischen Gleichgewichts) als Eckpunkte einer Sicherheitsratsreform durchsetzen. Die Diskussion blieb vielmehr von den nationalen Interessen der Staaten dominiert, in zweiter Hinsicht auch von regionalen Interessen.

4. Im Laufe der Jahre hat sich das weltpolitische Umfeld zum Nachteil des Reformvorhabens verändert. Der Optimismus der frühen 1990-er Jahre in Folge des Endes des Kalten Krieges und des die internationalen Beziehungen jahrzehntelang belastenden Ost-West-Gegensatzes ist gründlich verschwunden. Die westlichen Staaten, die, angeführt von den USA, nach dem Zweiten Weltkrieg die Vereinten Nationen als einen Eckstein des multilateralen Systems hervorgebracht haben, agieren global nicht mehr aus einer selbstbewussten Position ideologischer, wirtschaftlicher und militärischer Stärke. Die Vereinigten Staaten sehen sich mit den politischen und finanziellen Grenzen ihres Weltführungsanspruchs konfrontiert. Die wechselseitigen Spannungen zwischen den USA einerseits und Russland und China andererseits nehmen zu, ebenso die zwischen diesen drei Mächten und der Europäischen Union. Russland kämpft um den Erhalt seiner Weltmachtstellung. Gleichzeitig wächst die Enttäuschung der Entwicklungsländer Afrikas und Asiens darüber, dass das Wohlstandsgefälle zwischen ihnen und den nördlichen Industriestaaten auch in den letzten Jahrzehnten nicht kleiner geworden und insofern ihre jahrzehntelange Politik in den UN erfolglos geblieben ist.

5. Vor diesem Hintergrund zum Teil widersprüchlicher Veränderungen der internationalen Kräfteverhältnisse und des Staatensystems sind sich die Hauptakteure in der Reformdebatte ihrer künftigen Stellung und ihres „Auftrags“ nicht mehr sicher. Der weltpolitische Boden schwankt. In dieser Situation kann gegenwärtig von einem ,constitutional moment“63 (ähnlich dem des Jahres 1945), in dem sich durch Änderungen der UN-Charta als Weltverfassung die Koordinaten des internationalen Systems neu bestimmen lassen würden, nicht

61 Vgl. etwa Fassbender 1998a, S. 277 ff.: „A Constitutional Right of Veto“.

62 Vgl. hierzu Paulus 2001, S. 250 ff.

63 Vgl. für eine erste Verwendung dieses Begriffs im völkerrechtlichen Kontext Fassbender 1998a, S. 98 f. 
die Rede sein. Die Haltung zentraler Akteure (USA, Russland, Großbritannien, Frankreich) ist defensiv und auf den Erhalt ihrer bisherigen internationalen Stellung gerichtet, während sich China in Erwartung einer künftigen noch stärkeren Position abwartend verhält. Die Ansprüche der G 4-Staaten auf einen ständigen Sitz haben im Vergleich mit den 1990-er Jahren an Plausibilität verloren; vermutlich findet Indien als mit über 1,3 Milliarden Menschen zweitbevölkerungsreichstes Land der Erde noch die größte Unterstützung.

6. Aus völkerrechtlicher Sicht ist zu bemerken, dass eine grundlegende Reform des Sicherheitsrates und damit der UN als Ganzes eine in der Geschichte des modernen Völkerrechts einmalige Leistung gewesen wäre. Denn in dieser Geschichte ist bisher - vom Westfälischen Frieden über den Wiener Kongress bis hin zum Völkerbund und den Vereinten Nationen - eine wesentliche Neuordnung des Staatensystems stets das Resultat eines verlustreichen Krieges gewesen. Wer in diesem Krieg obsiegte, bestimmte die neue Ordnung oder suchte die alte wiederherzustellen. Damit verglichen wäre eine Reform des Sicherheitsrates im Wege vernunftgeleiteter, gemeinschafts- und zukunftsorientierter Diskussionen, Verhandlungen und Kompromisse in einem weltbürgerlichen Geist ein Ausweis der Reife der Menschheit am Beginn des 21. Jahrhunderts gewesen.

7. Das (jedenfalls vorläufige) Scheitern der Reform des Sicherheitsrates hat negative Rückwirkungen auf die im Zeichen der UN-Charta errichtete Völkerrechtsordnung. Denn, wie Christian Tomuschat jüngst bemerkt hat, die internationale Rechtsordnung hat durch die Charta und ihre Weiterentwicklung in der Praxis der Weltorganisation ,ein Normengerüst erhalten, das den Staaten in ihren internationalen Beziehungen feste Bindungen auferlegt, die sehr weitgehend die souveräne Handlungsfreiheit einschränken“. Auf diese Weise sei das Völkerrecht sehr viel verlässlicher und berechenbarer geworden, als dies in der Zeit vor 1945 der Fall gewesen sei, auch wenn sich die Durchsetzungs- und Sanktionsverfahren nach wie vor durch deutliche Schwächen auszeichneten. ${ }^{64}$ Der Sicherheitsrat aber ist das zentrale Durchsetzungs- und Sanktionsorgan der Völkerrechtsordnung der Vereinten Nationen, weshalb seine sukzessive Delegitimierung durch eine Reformunfähigkeit der UN auch die internationale Rechtsordnung beschädigen muss.

8. Wenn aus den oben skizzierten Gründen in der überschaubaren Zukunft auch nicht mit einer Reform der Zusammensetzung und der Abstimmungsregeln des Sicherheitsrates gerechnet werden kann, so wird die Reformdebatte doch fortgesetzt werden. Alle beteiligten Staaten und Staatengruppen haben im Verlauf von fast dreißig Jahren zu viel politische Kraft und Prestige investiert, um nun klein beizugeben. Das gilt sowohl für jene, die (wie die G 4-Staaten, die afrikanischen Staaten und die G 77-Entwicklungsländer) ihre Stellung im Sicherheitsrat zu verbessern suchten, als auch für die, die (wie die gegenwärtigen „P 5“ und die Uniting for Consensus-Staaten) gerade in einer Abwehr solcher Bemühungen eine Verteidigung ihrer internationalen Stellung gesehen haben. Die UN-Generalversammlung kann die von ihr jahrzehntelang geforderte Sicherheitsratsreform nicht ohne Ansehensverlust einfach aufgeben. So wurde die Reform schon am Beginn der 75. Sitzungsperiode von einer Reihe von Staats- und Regierungschefs, insbesondere afrikanischer Staaten, angemahnt. ${ }^{65}$ Auch die Politik- und die Völkerrechtswissenschaft werden sich mit dem Thema weiter beschäftigen, in welchem sich wie kaum einem anderen Grundfragen des Staatensystems und

64 Tomuschat 2020, 203.

65 Zusammenfassung der Reden: UNO, Generalversammlung 2020 g. 
der internationalen politischen und rechtlichen Ordnung bündeln. „The question of equitable representation on and increase in the membership of the Security Council", erklärte die Präsidentin der Generalversammlung in der 73. Sitzungsperiode, Botschafterin Espinosa Garcés (Ecuador), im Juni 2019, ,remains one of the most important, complex and sensitive processes before the General Assembly“ ${ }^{66}$ Ein Ende der Debatte ist nicht abzusehen.

\section{Bibliographie}

Fassbender, Bardo (1998a): UN Security Council Reform and the Right of Veto: A Constitutional Perspective. The Hague/London/Boston: Kluwer Law International.

Fassbender, Bardo (1998b): Reforming the United Nations. In: Die Friedens-Warte 73/4, S. 427-442.

Fassbender, Bardo (2003): All Illusions Shattered? Looking Back on a Decade of Failed Attempts to Reform the UN Security Council. In: Max Planck Yearbook of United Nations Law 7 (2003), S. $183-218$.

Fassbender, Bardo (2004): Pressure for Security Council Reform. In: Malone, David M. (Hrsg.), The UN Security Council: From the Cold War to the 21st Century. Boulder, Colorado/London: Lynne Rienner, S. 341-355.

Fassbender, Bardo (2005): On the Boulevard of Broken Dreams: The Project of a Reform of the UN Security Council after the 2005 World Summit. In: International Organizations Law Review 2 (2005), S. 391-402.

Fassbender, Bardo (2006): UN-Reform und kollektive Sicherheit. Der Bericht des ,UN High-level Panel on Threats, Challenges and Change' vom Dezember 2004 und die Empfehlungen des UNGeneralsekretärs vom März 2005. In: Heinrich-Böll-Stiftung (Hrsg.), Die Zukunft des Völkerrechts in einer globalisierten Welt. Baden-Baden: Nomos, S. 165-191.

Fassbender, Bardo (2012): The Security Council - Progress is Possible but Unlikely. In: Cassese, Antonio (Hrsg.), Realizing Utopia: The Future of International Law. Oxford: Oxford University Press, S. 52-60.

Fassbender, Bardo (2020): Key Documents on the Reform of the UN Security Council 1991-2019. Leiden/Boston: Brill Nijhoff.

Paulus, Andreas L. (2001): Die internationale Gemeinschaft im Völkerrecht. München: C. H. Beck.

Rittberger, Volker/Baumgärtner, Heiko (2005): Die Reform des Weltsicherheitsrats - Stand und Perspektiven. In: Die Friedens-Warte 80/3-4, S. 307-331.

Swart, Lydia (2013): Reform of the Security Council: 2007-2013. In: Freiesleben, Jonas von/Swart, Lydia (Hrsg.): Governing \& Managing Change at the United Nations: Security Council Reform from 1945 to September 2013. New York: Center for UN Reform Education, S. 23-59.

Tomuschat, Christian (2020): Konstitutionalisierung des Völkerrechts durch die Vereinten Nationen. In: Grothe, Ewald/Schlegelmilch, Arthur (Hrsg.), Constitutional Moments. Berlin: Berliner Wissenschafts-Verlag, S. 185-203.

\section{Dokumente der Vereinten Nationen}

UNO, Generalsekretär (2004): A more secure world: Our shared responsibility. Report of the High-level Panel on Threats, Challenges and Change. UN-Doc. A/59/565.

UNO, Generalsekretär (2005): In larger freedom: Towards development, security and human rights for all. Report of the Secretary-General. UN-Doc. A/59/2005.

66 UNO, Generalversammlung 2019a, S. 1. 
UNO, Generalversammlung (1991): Provisional Verbatim Record of the 68th Meeting, Agenda Item 38, Question of equitable representation on and increase in the membership of the Security Council. UN-Doc. A/46/PV.68, 11.12.1991.

UNO, Generalversammlung (1992a): Provisional Verbatim Record of the 69th Meeting, Agenda Item 40, Question of equitable representation on and increase in the membership of the Security Council. UN-Doc. A/47/PV.69, 23.11.1992.

UNO, Generalversammlung (1992b): Question of equitable representation on and increase in the membership of the Security Council. Resolution 47/62, UN-Doc. A/RES/47/62, 11.12.1992.

UNO, Generalversammlung (1993): Question of equitable representation on and increase in the membership of the Security Council. Resolution 48/26, UN-Doc. A/RES/48/26, 03.12.1993.

UNO, Generalversammlung (1995): Declaration on the Occasion of the Fiftieth Anniversary of the United Nations. Resolution 50/6, UN-Doc. A/RES/50/6, 24.10.1995.

UNO, Generalversammlung (1998): Question of equitable representation on and increase in the membership of the Security Council and related matters. Resolution 53/30, UN-Doc. A/RES/53/30, 23.11.1998.

UNO, Generalversammlung (2000): United Nations Millennium Declaration. Resolution 55/2, UNDoc. A/RES/55/2, 08.09.2000.

UNO, Generalversammlung (2005): 2005 World Summit Outcome. Resolution 60/1, UN-Doc. A/ $\mathrm{RES} / 60 / 1,16.09 .2005$.

UNO, Generalversammlung (2015): Declaration on the occasion of the seventieth anniversary of the United Nations. Resolution 70/3, UN-Doc. A/RES/70/3, 23.10.2015.

UNO, Generalversammlung (2019a): Agenda item 124 (continued): Question of equitable representation on and increase in the membership of the Security Council and other matters related to the Security Council. 92nd plenary meeting, UN-Doc. A/73/PV.92, 25.06.2019.

UNO, Generalversammlung (2019b): Agenda item 122: Question of equitable representation on and increase in the membership of the Security Council and other matters related to the Security Council. UN-Doc. A/74/PV.33, 33rd plenary meeting.

UNO, Generalversammlung (2019c): Agenda item 122: Question of equitable representation on and increase in the membership of the Security Council and other matters related to the Security Council. UN-Doc. A/74/PV.34, 34th plenary meeting, 25.11.2019.

UNO, Generalversammlung (2019d): Appointment of H.E. Ms. Lana Zaki Nusseibeh, Permanent Representative of the United Arab Emirates, and H.E. Ms. Joanna Wronecka, Permanent Representative of Poland to the United Nations, as Co-Chairs of the Intergovernmental Negotiations. Letter of the President of the General Assembly, 04.12.2019. https://www.un.org/pga/74/2019/12/04/ question-of-equitable-representation-on-and-increase-in-the-membership-of-the-security-counciland-other-matters-related-to-the-security-council-intergovernmental-negotiations/ (abgerufen am 03.09.2020).

UNO, Generalversammlung (2020a): Declaration on the Commemoration of the Seventy-fifth Anniversary of the United Nations. Final Draft. Letter of the President of the General Assembly, 03.07.2020, Annex (angenommen im Verfahren der silence procedure mit Wirkung vom 07.07.2020). https://www.un.org/pga/74/2020/07/03/final-draft-declaration-for-the-commemora tion-of-the-seventy-fifth-anniversary-of-the-united-nations-3/ (abgerufen am 03.09.2020).

UNO, Generalversammlung (2020b): Security Council Reform. Statement by H.E. Tijjani Muhammad Bande, President of the 74th Session of the United Nations General Assembly, 13.02.2020. https://www.un.org/pga/74/2020/02/13/security-council-reform-2/ (abgerufen am 03.09.2020).

UNO, Generalversammlung (2020c): Letter dated 24 February 2020, from the co-chairs of the Intergovernmental Negotiations on the question of equitable representation on and increase in the 
membership of the Security Council and matters related to the Security Council (IGN). Annex to a letter dated 24 February 2020 of the President of the General Assembly, 24.02.2020. https:// www.un.org/pga/74/2020/02/24/security-council-reform-3/ (abgerufen am 03.09.2020).

UNO, Generalversammlung (2020d): Letter dated 7 May 2020, from the co-chairs of the Intergovernmental Negotiations on the question of equitable representation on and increase in the membership of the Security Council and matters related to the Security Council (IGN). Annex to a letter dated 8 May 2020 of the President of the General Assembly, 8.05.2020. https://www.un.org/ pga/74/2020/05/08/security-council-reform-5/ (abgerufen am 03.09.2020).

UNO, Generalversammlung (2020e): Letter dated 29 July 2020, from the co-chairs of the Intergovernmental Negotiations on the question of equitable representation on and increase in the membership of the Security Council and matters related to the Security Council (IGN). Annex to a letter dated 30 July 2020 of the President of the General Assembly, 30.07.2020. https://www. un.org/pga/74/2020/07/30/security-council-reform-6/ (abgerufen am 03.09.2020).

UNO, Generalversammlung (2020 f.): Draft decision entitled „Question of equitable representation on and increase in the membership of the Security Council and other matters related to the Security Council“" (A/74/L.88). Letter from the President of the General Assembly, 31.08.2020. https://www.un.org/pga/74/2020/08/31/question-of-equitable-representation-on-and-increasein-the-membership-of-the-security-council-and-other-matters-related-to-the-security-council/ (abgerufen am 03.09.2020). Text der Draft decision, 26.08.2020: https://www.un.org/ pga/74/2020/08/26/pga-letter-on-the-draft-decision-entitled-question-of-equitable-representati on-on-and-increase-in-the-membership-of-the-security-council-and-other-matters-related-to-thesecurity-council/ (abgerufen am 03.09.2020).

UNO, Generalversammlung (2020 g): Plenary Meetings Coverage, Seventy-fifth session, 3rd meeting. GA/12267, 21.09.2020. https://www.un.org/press/en/2020/ga12267.doc.htm (abgerufen am 29.10.2020). 


\section{Die Friedens-Warte}

Journal of International Peace and Organization

2020 | Vol. 93

\section{Herausgeber}

Prof. Dr. iur. Andreas von Arnauld

Walther-Schücking-Institut für Internationales Recht

Christian-Albrechts-Universität zu Kiel

Westring 400 | 24118 Kiel

arnauld@wsi.uni-kiel.de

Dr. rer. pol. Charlotte Dany

Friedensakademie Rheinland-Pfalz

Akademie für Krisenprävention und zivile Konflikt-

bearbeitung | Universität Koblenz-Landau

Kaufhausgasse 9|76829 Landau

dany@uni-landau.de

Prof. Dr. phil. Michael Staack

Institut für Internationale Politik

Helmut-Schmidt-Universität/

Universität der Bundeswehr Hamburg

Holstenhofweg 85 | 22043 Hamburg

michael.staack@hsu-hh.de

Prof. Dr. iur. Pierre Thielbörger

Institut für Friedenssicherungsrecht

und Humanitäres Völkerrecht (IFHV)

Ruhr-Universität Bochum

Massenbergstraße 9 B | 44787 Bochum

pierre.thielboerger@rub.de

\section{Redaktion}

Prof. Dr. iur. Andreas von Arnauld (v. i.S.d.P.)

Walther-Schücking-Institut für Internationales Recht

Christian-Albrechts-Universität zu Kiel

Westring 400 | $24118 \mathrm{Kiel}$

arnauld@wsi.uni-kiel.de

Redaktionsassistenz Sonja Dietz, LL.B.

Namentlich gekennzeichnete Beiträge geben die persönliche Meinung des Verfassers wieder und stellen nicht unbedingt die Auffassung der Redaktion dar

\section{Beirat}

Günter Bierbrauer (Osnabrück) | Thorsten Bonacker (Marburg) | Tanja Brühl (Darmstadt) | Michael Brzoska (Hamburg) | Sven Chojnacki (Berlin) | Jost Dülffer (Köln) | Martina Fischer (Berlin) | Wolff Heintschel v. Heinegg (Frankfurt/O.) | Harald Müller (Frankfurt/M.) $\mid$ Georg Nolte (Berlin/Den Haag) | Stefan Oeter (Hamburg) $\mid$ Andreas L. Paulus (Göttingen/Karlsruhe) | Anne Peters (Heidelberg) | Thilo Rensmann (Augsburg) | Ulrich Schneckener (Osnabrück) | Eva Senghaas-Knobloch (Bremen) $\mid$ Christian Tomuschat (Berlin)

\section{Peer Review}

Die Friedens-Warte ist ein refereed journal. Eingereichte Beiträge unterliegen einem Begutachtungsverfahren, das über die Aufnahme in die Zeitschrift entscheidet

\section{Homepage}

friedenswarte.bwv-verlag.de elibrary.bwv-verlag.de/journal/fw

\section{Erscheinungsweise}

Zweimal jährlich als Doppelheft

\section{Bezugsbedingungen}

Bestellung beim Verlag und durch jede Buchhandlung. Bezugspreise für Privatpersonen: Abonnement Print jährlich $78,-€$ (ermäßigt 60,- $€$ ) $\mid$ Abonnement Print + Digital jährlich 103,- $€$ (ermäßigt 85,- $€$ ) | Einzelheft $25,20 € \mid$ Dop- pelheft 45,- $€$. Bezugspreise für Institutionen: Abonnement Print 85,40€| Abonnement Print + Digital jährlich (mit IPZugang) $185,70 € \mid$ Einzelheft 25,20 $€ \mid$ Doppelheft 45,- $€$. Alle Preise inkl. MwSt., zzgl. Portokosten für die Printausgabe (Jährliches Abonnement: Inland 9,80€| Ausland 19,00€ Einzelheft: Inland $3,95 € \mid$ Ausland 7,50€. Direktliefergebühr: $2,-€$.) Irrtum und Preisänderung vorbehalten.

Die Mindestabonnementdauer beträgt ein Jahr. Die Bezugszeit des Abonnements verlängert sich automatisch um ein weiteres Jahr, falls das Abonnement nicht sechs Wochen vor Ablauf des Kalenderjahres schriftlich beim Verlag gekündigt wird.

Zuschriften, die den Vertrieb oder Anzeigen betreffen, bitte nur an den Verlag.

\section{Verlag}

BWV $\mid$ Berliner Wissenschafts-Verlag GmbH

Behaimstraße $25 \mid 10585$ Berlin

Tel. +49 (0)30841770 0 | Fax +49 (0) 3084177021

bwv@bwv-verlag.de

www.bwv-verlag.de

\section{Layout und Herstellung}

Maria Ostrowski | ostrowski@bwv-verlag.de

\section{Satz}

DTP+TEXT Eva Burri, Stuttgart

\section{Anzeigen}

Franziska Fiebig (verantwortlich)

BWV | Berliner Wissenschafts-Verlag GmbH

Behaimstraße 25 | 10585 Berlin

marketing@bwv-verlag.de

Tel. +49 (0) 3084177026

Die Mediadaten sind abrufbar unter

www.bwv-verlag.de

\section{Druck und Verarbeitung}

docupoint, Magdeburg

\section{Urheber- und Verlagsrechte}

Alle in dieser Zeitschrift veröffentlichten Beiträge sind urheberrechtlich geschützt. Jede Verwertung in anderen als den gesetzlich zugelassenen Fällen bedarf der vorherigen schriftlichen Einwilligung des Verlages.

Mit der Annahme zur Veröffentlichung überträgt der Autor dem Verlag das ausschließliche Verlagsrecht bis zum Ablauf des Urheberrechts. Eingeschlossen ist auch das Recht zur Herstellung elektronischer Versionen und zur Aufnahme in Datenbanken sowie das Recht zur Verbreitung und Vervielfältigung online oder offline ohne zusätzliche Vergütung. Vgl. dazu auch die Angaben unter https://www. bwv-verlag.de/selbstarchivierungself-archiving.

Alle Anfragen und Manuskriptangebote bitte an die Adresse der Redaktion. Für unverlangt eingesandte Manuskripte wird keine Haftung übernommen.

Der Berliner Wissenschafts-Verlag beachtet die Regeln des Börsenvereins des Deutschen Buchhandels e.V. zur Verwendung von Buchrezensionen. Keine Garantie für Besprechung oder Rückgabe bei unverlangt eingesandten Rezensionsstücken.

(C) Berliner Wissenschafts-Verlag, Berlin 2020

Printed in Germany.

ISSN (Print) 0340-0255

ISSN (Online) 2366-6714

Die Friedens-Warte im Internet unter www.bwv-verlag.de 


\section{Die Friedens-Warte}

\section{F-W}

Vol. 93 | 2020 | Issue 3-4
ISSN-Print $\quad$ 0340-0255

ISSN-Online 2366-6714

\section{Geleitwort der Herausgeber I \\ A Note from the Editors}

Editorial: Ein Dank an Christian Tomuschat I

Editorial: Thanking Christian Tomuschat

\section{Beiträge I Articles}

\section{Menschenrechte I Human Rights}

"It Is Not Possible For Us That Injustice Be Justice". Some

Remarks on the Soghomon Tehlirian Trial at Age 100

DANIEL-ERASMUS KHAN

\section{Europäischer Menschenrechtsschutz in der Krise?}

Die COVID-19-Pandemie als Herausforderung und Chance CHRISTINA BINDER

Verlust der Staatsangehörigkeit als Maßnahme der Terrorismusbekämpfung: Welche Grenzen setzt das Völkerrecht? CHRISTIAN WALTER/PHILIP NEDELCU

\section{Internationale Organisation und}

\section{Friedenssicherung I International}

Organisation and Peacekeeping

Strindberg, Fried und Tomuschat: Internationale Gerichte

und bewaffnete Konflikte

CHRISTIAN J. TAMS

The Creation of the United Nations: Break with the Past

or Continuation of Wartime Power Politics?

OLIVER DIGGELMANN
Die Beharrungskraft des Status quo: Die Bemühungen um eine Reform des Sicherheitsrates im Jahr des 75-jährigen Bestehens der Vereinten Nationen

BARDO FASSBENDER

Military Assistance Based on Ex-Ante Consent: a Violation of Article 2 (4) UN Charter?

ERIKA DE WET

\section{Debatte I Debate}

Für Stabilität und gegen unkontrollierbare Konfrontation - eine politische Initiative zu Dialog und Zusammenarbeit mit Russland auf der Grundlage einer glaubwürdigen europäischen und transatlantischen Sicherheitspolitik

RÜDIGER LÜDEKING/HELMUT W. GANSER

\section{Panorama I Panorama}

\section{Konferenzbericht I Conference Report}

Expert Roundtable Discussion: Formalisation Processes

in Extractive Sectors as Part of State- and Peacebuilding

Strategies - Peace Academy Rhineland-Palatinate, University

of Koblenz-Landau, 27-28 February 2020

ZABRINA WELTER/CHRISTINA ANKENBRAND

\section{Buchbesprechung I Book Review}

Jorge E. Viñuales (ed.), The UN Friendly Relations

Declaration at 50

BENEDIKT BEHLERT 